\title{
Harnessing the Benefits of Bimanual and Multi-finger Input for Supporting Grouping Tasks on Interactive Tabletops
}

\author{
Florian Geyer, Anita Höchtl, Harald Reiterer \\ Human-Computer Interaction Group, University of Konstanz \\ Universitätsstraße 10, 78457 Konstanz, Germany \\ \{firstname.lastname\}@uni-konstanz.de
}

\begin{abstract}
In this paper we describe an experimental study investigating the use of bimanual and multi-finger input for grouping items spatially on a tabletop interface. In a singleuser setup, we compared two typical interaction techniques supporting this task. We studied the grouping and regrouping performance in general and the use of bimanual and multi-finger input in particular. Our results show that the traditional container concept may not be an adequate fit for interactive tabletops. Rather, we demonstrate that informal and organic spatial metaphors are able to harness more benefits of multi-finger and bimanual interaction. We conclude with recommendations for the design of grouping techniques on interactive surfaces.
\end{abstract}

\section{Author Keywords}

Grouping; Interactive Tabletops; Multitouch, Metaphors

\section{ACM Classification Keywords}

H.5.2. User Interfaces: Input devices and strategies (e.g., mouse, touchscreen)

\section{General Terms \\ Design, Experimentation, Human Factors}

\section{INTRODUCTION}

Arranging and organizing digital objects is a common task in various application domains. From sorting photos to managing files, many activities require users to move single or multiple items around for creating spatial aggregations or groups. The manual way of moving and dragging objects thereby serves as an implicit tool for filtering and synthesizing, thereby taking advantage of the human capabilities of recall, visual search and reasoning [5].

Multi-touch interaction bears great potential for supporting the manipulation of individual items and groups more efficiently than it is possible with traditional single pointer desktop interfaces. Further, when the mapping between gesture and action becomes more direct, users can make more use of their spatial memory capabilities and it is easier to move and remember objects [6]. Nevertheless, due to the rich affordances of touch interfaces, it is possible to manipulate virtual objects and groups of objects with not only one hand, but with two hands and multiple fingers, thereby making interaction more analogous to physical interactions in the non-digital world.

Researchers have examined the characteristics of digital and physical affordances [8], gestures for typical tasks and commands [11] and bimanual gestures for moving and grouping items $[9,6]$. In our research, we build upon this work for examining the use of multi-touch interaction techniques for spatial grouping and re-grouping tasks. In previous work, we compared a container and a proximity technique in regard to task efficiency, user preferences and interaction strategies [3]. In this paper, we focus on the use of bimanual and multi-finger input in both interaction techniques and their implications for designing natural user interfaces.

\section{RELATED WORK}

Our research is related to studies of bimanual multitouch interaction in general as well as interaction techniques for supporting grouping tasks on interactive surfaces in particular. Terrenghi et al. [8] have explored differences in affordances with manipulation of digital content on interactive surfaces and traditional physical interactions. They make the point that "in order to confer the benefits of bimanual interaction, one approach is to design specific tools and techniques which more explicitly require asymmetric bimanual interaction" [8]. Wobbrock et al. [11] and North et al. [6] also show that two-handed interaction forms an important part of surface gesture design. Because there is a vast body of other work in bimanual interaction, we refer to Kin et al. [4] for an overview of studies in multifinger and bimanual gestures on touch interfaces.

Besides the investigation of grouping gestures, researchers have also created interaction techniques especially for grouping items on tabletops. Traditionally, multi-selection and grouping tasks on desktop interfaces are supported by group selection methods such as rubber banding or lasso selection, before operations (e.g. move into container) are applied to the selected group [10]. This method of group selection and drag \& drop style operations was also carried over to interactive surfaces. Examples for this can be found in research and practice [7,2] and are now supported by most non-commercial and commercial frameworks such as 
Surface SDK. Some innovative concepts based on containment are interaction techniques based on piles or multimodal selection and grouping based on gestures and speech [9].

\section{CONTAINMENT VS. PROXIMITY}

Our literature review revealed that the container concept can be considered as state of the art not only on desktop systems but also on interactive touch surfaces. We also found empirical evidence that there is considerable potential in designing for bimanual and multi-finger input. However, the container concept was not intentionally designed for such informal and physical interactions. Hence, we see a need for investigating grouping techniques that may better harness the potential benefits of multitouch and bimanual interaction. In search for alternative grouping concepts, we were inspired by Watanabe et al.'s interaction technique called "Bubble Clusters" [10] for grouping items on a mouse-operated desktop system. Their study showed that the technique could improve grouping performance in a simple single-pointer icon relocation task compared to standard folders and lasso selection. We hypothesize that such informal grouping techniques based on spatial proximity might be closer to our natural physical interactions and that these might be better suitable for use on interactive tabletops. Therefore, we think that it is valuable to explore an adaptation of this mouse-based grouping technique for use on multitouch displays.

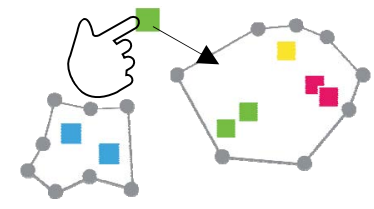

Figure 1: Bin: grouping items by dragging into containers.

\section{The Bin Technique}

We designed a containment metaphor "Bin" based on "Storage Bins" [7]. This container concept for tabletops can be considered as an equivalent to folders on desktop interfaces. Items can be dragged into movable and resizable containers. Once dragged into the container, they are scaled down as to allow for space-efficient organization. Yet, the classical concept of containment was adapted by including eight handles which allow adjusting the bin's shape (see Figure 1). Users can add items to the bin and may freely arrange them inside the bin. One may also move the bin itself by dragging it to a new location. A bin provides several ways of interaction as described in the following: 1) Dragging objects into a bin: The user may either drag or toss an object directly into a bin. When the item is released inside a bin, it is resized as to show that it is now contained by the bin. Users may also select a group of objects with a lasso selection and may then move this collection into the bin. After releasing the collection, objects are added to the bin. 2) Collecting objects: The bin itself can also be used to collect items. Therefore, users can drag the bin directly over objects or adjust the shape of the bin by dragging its handles. After releasing the bin or its handles, objects inside the bin's boundaries are resized to visualize containment.

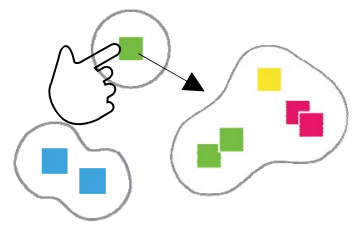

Figure 2: Bubble: grouping items by proximity.

\section{The Bubble Technique}

For the proximity metaphor we adapted the interaction technique "Bubble Clusters" [10] for use on interactive tabletops. Therefore, each object is surrounded by a bubble, which adjusts its boundaries according the number and positions of objects in close proximity (see Figure 2). Our multi-touch adaptation "Bubble" provides following interaction techniques for organizing items: 1) Group by object: Two items join one bubble if they are positioned close to each other. To do so, the user drags an object to another object and as soon as their boundaries touch each other, bubbles melt, creating a new, larger bubble. 2) Group by bubble: Users can also move bubbles by dragging the bubble shape to a new position. After releasing it, overlapping items or other bubbles are merged, when applicable. 3) Splitting a bubble: Users may also split a bubble by drawing a stroke with the finger across the bubble shape. The bubble then splits and two new bubbles result.

\section{USER STUDY}

We conducted a user study to compare both concepts regarding differences in performance and bimanual as well as multi-finger input. Eventually, by combining both findings, we wanted to examine whether the Bubble technique as an organic concept allows for harnessing more of the benefits of multi-touch input for grouping tasks.

\section{Procedure \& Tasks}

Our controlled experiment was performed in our lab on a Microsoft Surface table (24" x 18”, 1024 x 768 px). Questionnaires, structured interviews, video-recordings and logging data were collected for data analysis. Twelve students (seven male and five female, age 21 to 39, mean: 26) were recruited for the study from the local university campus. We further used a counterbalanced within-subjects design whereby each condition was introduced to the participants prior to each test run by a description supplied on a paper sheet. Each condition was tested in sessions that lasted approximately 20 minutes. The whole procedure took around 60 minutes.

Users were given the task to group and regroup 30 colored rectangles into clusters according to five different colors. In 


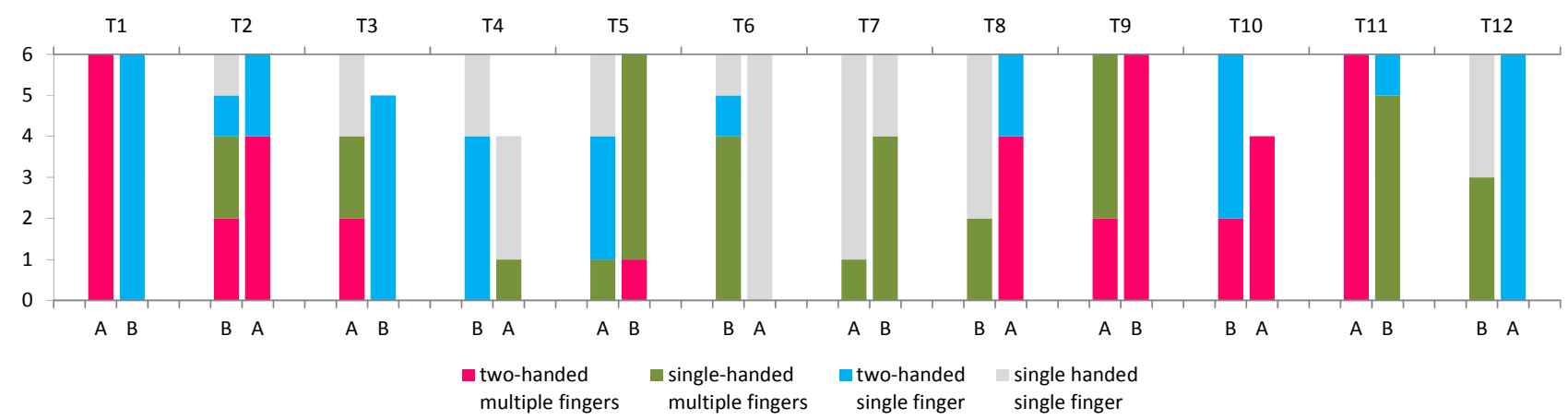

Figure 3: Participant's use of bimanual and multi-finger interaction in trials with Bubble (A) and Bin (B).

order to avoid carryover effects, six participants started with the Bin and the other six started with the Bubble condition. Each task covered a grouping and a regrouping phase: First, the rectangles appeared distributed randomly on the display. Then, users were asked to group shapes freely according to their colors. After a five second break, the colors of the shapes were shuffled and participants had to regroup the objects again. Compared to the grouping phase, the rectangles were now already clustered, forcing a regrouping. The whole task procedure consisted of 16 trials ( 4 runs $\times 2$ interface conditions $\times 2$ phases), whereby the first run was practice and was excluded from data analysis.

\section{Data Analysis}

Our data analysis focused on the differences both concepts yield in general performance and the use of bimanual and multi-finger input in particular.

One of our research goals was to find out, how participants completed the grouping task with both interfaces. This was measured by comparing the task completion times of each trial. Paired t-tests for the grouping phase depicted significant differences between both techniques $(\mathrm{t}(12)=$ $0.00063, \mathrm{p}<0.001)$ with the Bubble technique being faster (avg. 54 seconds) than the Bin technique (avg. 87 seconds). Measured times for the regrouping phase however show only slight variations.

We analyzed our data as to investigate the differences concerning bimanual and multi-touch input between the interfaces. Therefore, 71 successful trials with the Bin technique and 68 successful trials with the Bubble technique were qualitatively analyzed using the video material. Each trial of both conditions was coded based on the use of one-handed and two-handed interaction as well as single finger and multiple finger interaction. Interactions such as lasso selection and bubble splitting which explicitly require the use of multiple fingers or both hands were excluded from this analysis. Figure 3 depicts the results of this analysis for all trials in order of occurrence in the user study. None of the participants (T1 to T12) had one preferred way of interacting across both interfaces. Only one subject (T1) kept to one interaction strategy in both conditions. Most participants adapted their interaction between the trials and between grouping and regrouping phases. Four participants (T3, T6, T9 and T12) tried different combinations in the first interface, but eventually decided for one strategy in the second condition. In contrary, one participant (T11) tried different strategies in the second condition only. Overall, all subjects tried different combinations between two patterns $(\mathrm{N}=4)$, three patterns $(\mathrm{N}=4)$ or all four patterns $(\mathrm{N}=4)$.

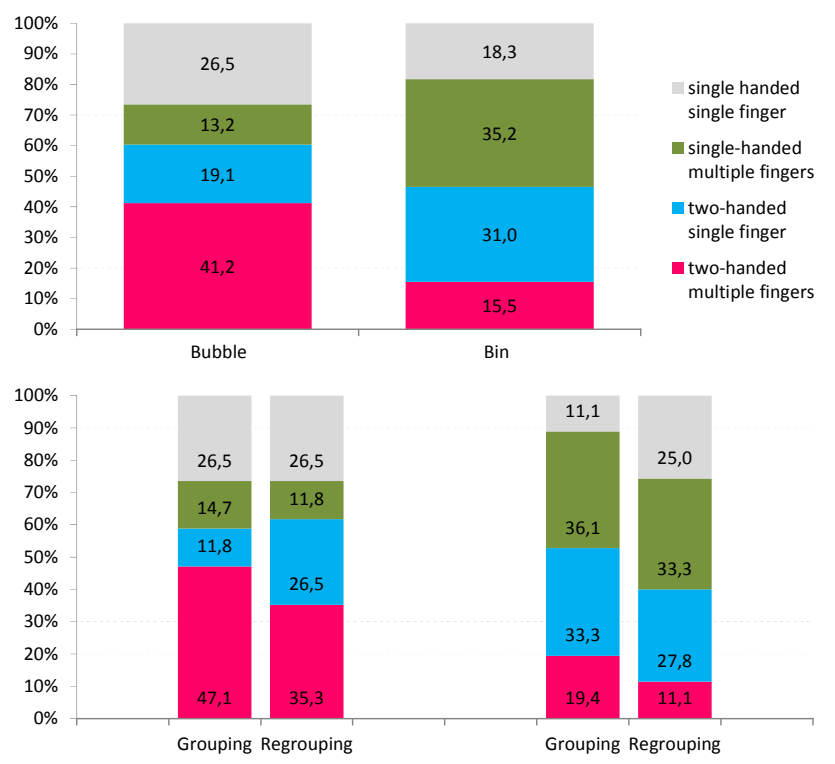

Figure 4: Interaction patterns with one or both hands and one or multiple fingers in the grouping and regrouping phases.

Figure 4 shows the percentages of interaction patterns for both interfaces. Two-handed interaction with multiple fingers was especially applied in the Bubble condition $(41.2 \%)$ whereas one hand and multiple fingers $(35.2 \%)$ as well as both hands with one finger each (31\%) was more often used in the Bin condition (Figure 4, top). Our analysis also revealed that participants used multiple fingers more often in the grouping phase than in the regrouping phase in both conditions. Bimanual use of multiple fingers was used most often (47.1\%) in the grouping phase of the Bubble condition (Figure 4, bottom). 


\section{Discussion}

Our results show that there are significant differences in performance within the grouping phase as the Bubble technique clearly outperformed the Bin technique. Our qualitative analysis indicates that this is at least partly due to increased use of bimanual interaction in combination with multiple fingers. However, we could not observe this behavior in the regrouping phase. One possible explanation for this is that the grouping phase more closely resembles tasks that are prevalent in everyday life (like sorting randomly distributed photos or cards), while the regrouping task was more artificial (sorting based on existing groups) [5]. Likewise, participants could make more use of their natural behavior in the grouping task. Participants also confirmed that the Bubble technique is more natural and easier to understand. We think that this is due to the difference in affordance both interfaces yield. While the Bin technique seems to make users carry over singlepointer interaction strategies from the desktop-world, the Bubble technique is entirely different, not pointing the users to the reuse of existing strategies. For example, we could identify a particular efficient strategy of moving multiple dispersed objects with both hands to a central location. Although this strategy is also possible in the Bin condition, participants applied it mostly in the Bubble condition. We think that this is due to the organic nature of the Bubble interface, which emphasizes merging operations rather than drag \& drop and that allows to make more flexible use of space for planning and conducting grouping tasks.

\section{CONCLUSION}

In this paper we compared the container and the proximity concept for supporting grouping tasks on interactive surfaces. The findings of our study showed that the proximity concept is more beneficial for grouping tasks on a digital surface than the traditional container concept. Participants interacted more often with multiple fingers and both hands, which depicts that grouping based on proximity is capable of harnessing more advantages of bimanual and multi-touch interaction than grouping based on containment. However, the validity of our findings is limited in the sense that we did not study long-term usage of a large user population. Results may differ based on the prior experiences of users and the use of both interaction techniques over longer periods of time.

The results of our study contribute to the design of interfaces for interactive surfaces in general and for specific task support in a wide range of work domains [5]. We consider the Bubble technique suitable for informal sensemaking tasks, but less for layout tasks as it does not preserve spatial positioning. Our findings also contribute to the understanding of metaphors and affordances on interactive surfaces. One the one hand, our study demonstrates that organic interface metaphors have great potential for harnessing the benefits of multi-touch interaction. On the other hand, we presented an example for the need to critically explore and adapt interaction techniques from the desktop before porting them to touch interfaces. We further see potential of extending and improving the Bubble technique not only for single users, but also for supporting multiple users and collaborative work, such as systems supporting creative group activities like brainstorming or affinity diagramming [1].

\section{ACKNOWLEDGEMENTS}

This research is funded by the German Research Foundation DFG (Deutsche Forschungsgemeinschaft) under grant number RE 1843/3-1.

\section{REFERENCES}

1.Geyer, F., Pfeil, U., Höchtl, A., Budzinski, J. and Reiterer, H. Designing reality-based interfaces for creative group work. In Proc. of C\&C 2011. ACM Press (2011), 165-174.

2.Hinrichs, U., Carpendale, S., Scott, S. D. and Pattison, E. Interface currents: supporting fluent collaboration on tabletop displays. In Proc. of SmartGraphics 2005, Springer (2005), 185197.

3.Höchtl, A., Geyer, F. and Reiterer, H. A comparison of spatial grouping techniques on interactive surfaces. In Proc. of Mensch \& Computer 2012, Oldenbourg (2012).

4.Kin, K., Agrawala, M. and DeRose, T. Determining the benefits of direct-touch, bimanual, and multifinger input on a multitouch workstation. In Proc. of GI 2009, Canadian Information Processing Society (2009), 119-124.

5.Kirsh, D. The intelligent use of space. In Artificial Intelligence Vol. 73 Iss. 1-2, Elsevier (1995), 31-68.

6.North, C., Dwyer, T., Lee, B., Fisher, D., Isenberg, P., Robertson, G. and Inkpen, K. Understanding multi-touch manipulation for surface computing. In Proc. of INTERACT 2009, Springer (2009), 236-249.

7.Scott, S. D., Carpendale, M. S. T. and Habelski, S. Storage bins: mobile storage for collaborative tabletop displays. Computer Graphics and Applications, Vol. 25 (4), IEEE Computer Society Press (2005), 58-65.

8.Terrenghi, L., Kirk, D., Sellen, A., and Izadi, S. Affordances for manipulation of physical versus digital media on interactive surfaces. In Proc. of CHI 2007, ACM Press (2007), 1157-1166.

9.Tse, E., Greenberg, S., Shen, C., Forlines, C., and Kodama, R. Exploring true multi-user multimodal interaction over a digital table. In Proc. of DIS 2008, ACM Press (2008), 109-118.

10.Watanabe, N., Washida, M., and Igarashi, T. Bubble clusters: an interface for manipulating spatial aggregation of graphical objects. In Proc. of UIST 2007, ACM Press (2007), 173-182.

11.Wobbrock, J. O., Morris, M. R., and Wilson, A. D. Userdefined gestures for surface computing. In Proc. CHI 2009, ACM Press (2009), 1083-1092. 